\title{
WAVE FUNCTION TO PARTITION FUNCTION: A CORRELATION
}

\author{
( Jaydip Datta )
}

In this note the statistical thermodynamics is correlated to wave function through mathematical probability. The note can be subdivided into two following portions.

Keywords:

Randomisation, Wave Function, Probability Density Function, Multiplicative Probability, Partition function, Correlation ( K.P ) coefficient, Probability , Weights, Stirring's Approximation, Boltzmann, Sigmoid function .

1. STATISTICS -THE BASIC OF STATISTICAL THERMODYNAMICS: A CORRELATION - It is analysed by linear regression method by Pearson coefficient, In our earlier Review some descriptive statistical features like linear regression aspects of Karl Pearson Coefficient / Correlation coefficient (1) has been studied specially. But the knowledge of Basic Statistics is the foundation course of STATISTICAL THERMODYNAMICS. The significant Correlation ( $R^{\wedge} 2$ lies between $0.2-0.85$ ) is nothing but the measure of PROBABILITY . Thus the Thermodynamic Probability (T.P) is a function of Weights of the Configuration (W ) (2) Stirling's approximation is also a good convergence with T.P as well randomization aspects of Entropy or Weights of Configuration (W) .

2. PROBABILITY : A THE BASICS OF WAVE FUNCTION - The term probability widely used in statistics is a measure of of spontaneity of a system . In this note we have an attempt to correlate the Probabilistic approach between the wave functional concept NORMALIZATION and the algebraic relation of the term probability .

\section{NORMALISATION OF PARTITION FUNCTION:}

In this report we wish to report a most probable approach of Normalisation on Partition function and also the nature of thermal wave function as a normalised parameter. But the main duality / paradox / is shi ( wave function ) is an intensive property depends on electronic nature But the Molecular wave function / Thermal wave function is a molecular property rather distribution so it is an extensive property . Now our attempt is to correlate the two properties through a mathematical theorem ie NORMALISATION. 
ALGEBRAIC THEORY OF PROBABILITY:

$\mathbf{P}(\mathbf{A})+\mathbf{P}(\mathbf{B})=\mathbf{P}(\mathbf{A}$ * $\mathbf{B})-$ Since Probability is always multiplicative Eqn ( 1 )

Here $\bmod [\mathrm{Shi}]^{\wedge} \mathbf{2}$ is known as probability density function of an electronic orbital

. Applying algebraic equation of Probability,

Shi [ A ] + Shi [ B ] = Shi [A ] * shi [ B ]----------Eqn ( 2 ) I By Comparing Eqn ( 1 ) \& ( 2 ) Shi [ A ] +

Shi $[B]=$

[ Shi ]^2-------- Eqn ( 3 )

Equation ( 3 ) is the Conclusive Correlated Equation. So greater is the [ Shi $]^{\wedge} \mathbf{2}$ greater is the Probability .

3. BOLTZMANN - SIGMOIDAL statistics - Extended part of random coil model .

\section{REFERENCES :}

1. Jaydip Datta , Statistical Correlation on Physico Chemical Analysis - An Integrative Teaching Approach , Statistical Correlation on Physico Chemical Analysis - An Integrative Teaching Approach , DOI: 10.2139/ssrn.4067432 .(

https://www.researchgate.net/publication/360240497 Statistical Correlation on Physico Ch emical_Analysis_-

An_Integrative_Teaching_Approach?_sg\%5B0\%5D=9DSLiUUV2pTvmiB5QdkPG5enbDF u8RTVF9JyFDOOOKQHyt8yXDmuc6rtaNwXfNJTghqxEnnuHncaQqbTLX6XcAT2UDHY fuAGyQn4xydT.1AU7xVu0OybZLf7r1FRt_chFejvg6LMws5oQd2wozZiXTf4TLG2m_sgsF 6WLUT-0PtCU5f9qRRigqU71HUk2rQ ).

2. Jaydip Datta , MATHEMATICAL STATISTICS - PART2 ,January 2022 ,DOI:

\section{$\underline{10.31219 / \mathrm{osf} . i 0 / 958 \mathrm{fm}}$.}

(https://www.researchgate.net/publication/335223319_MATHEMATICAL_STATISTICS

_PART2?_sg\%5B0\%5D=zNPA7TqcpHRRgTLchiq8iKqsEU2kaky92Kxn6oufgzMcSTm81 lhqdAm-ephUvhboB7yKdvI30UGluoot8Jzf9tdJ8YAgX-

jbdOlosNR0.6RG11rpJG6xUe_f3B-JD8t-ugERuPjrNud4AzorUqvjKc-

V CiikrpE8u00v3xLp-PUonmq6HR55d-DFOTijeA ).

DATTA, J. (2020, September 9). MATHEMATICAL STATISTICS - PART2.

https://doi.org/10.31219/osf.io/958fm

DATTA, JAYDIP. "MATHEMATICAL STATISTICS - PART2." OSF Preprints, 9 Sept. 2020. Web.

DATTA, JAYDIP. 2020. "MATHEMATICAL STATISTICS - PART2." OSF Preprints.

September 9. doi:10.31219/osf.io/958fm. 
3. JAYDIP DATTA et al, THE TRENDS OF MODERN PHYSICAL CHEMISTRY: A DISCLOSURE ,March 2022 ,DOI: 10.6084/m9.figshare.12263126.v9 ,License, ,CC BY-ND 4.0.

(https://www.researchgate.net/publication/340579762 THE TRENDS OF MODERN PHYSICAL CHE MISTRY A DISCLOSURE? sg=Qy66PplukW6amUnlOo2AWogLcNoXx9W29bH7Oh7WwwBp5V6ZY8 qaq1tUodC9U564v4acdEudqRyADMO6g9hcoOcmdKV8rdk5eL51L.BXQaDt7lw5F6WkPJ2SRA7e0isdXPa6v5OUyofApf5F2GfC6ISOWiKXgNI6szAm68hPZZxy w4KRXGHKyOpoiJtA )

4. JAYDIP DATTA, STATISTICS -THE BASIC OF STATISTICAL THERMODYNAMICS: A CORRELATION ( REVISED ) ,October 2021 ,DOI: 10.13140/RG.2.2.27055.05282/3 .

(https://www.researchgate.net/publication/339483700 STATISTICS -

THE BASIC OF STATISTICAL THERMODYNAMICS A CORRELATION REVISE $\mathrm{D} ? \mathrm{sg}=\mathrm{BvYHpE}$ C0Gnili7Yjcdk MAI7RQGf1OoPchfjyP3bnuw6ICAaXVUohyUOlfgaB0M7oaE5VHbk1IQRXU1epVFQC kE5rbNa908wOJQW-Ta.kHhgXKgPIUg48WSFNsgDErRaZGjkqF6 F6RKGjUV6MnLpSF1yUHYjbZ1953C6S2I8ObXsig67NKaNDGwRrg A ).

5. JAYDIP DATTA , A SEMINAR ON PHYSICAL CHEMISTRY TEACHING - SOME HIGHLIGHTS ,July 2020 ,DOI: 10.13140/RG.2.2.19024.46082/2 .

https://www.researchgate.net/publication/334327584 A SEMINAR ON PHYSICAL CHEMISTRY T EACHING -

SOME HIGHLIGHTS? sg=csanySlym3B6Qcap8k1h6ipjSiymwPpXsqzpzyDTNJv0Yr25Di5a3mRfTfR5ekEyg6Bo QY5pOmPbHUu3Ok9Z7J i8IRvmrM5vad8wQ.mLfYCd87UstBzCDwzGUqCVuxfo.Jp6lv\|lICnwzsMBzTeX0CWrJK3Y3ylAqQEGi0bhoJpUN7jVn-8AXUIcyXIWw )

6. Jaydip Datta, APPLICATION OF LINEAR REGRESSION ON STERN VOLMER EQUATION

March 2019 ,DOI: 10.13140/RG.2.2.16842.93120/1 ,License CC BY-SA 4.0 .

(https://www.researchgate.net/publication/331923759 APPLICATION OF LINEAR REGRESSION ON STERN VOLMER EQUATION? sg=W1uibsRfvmgSytEKvE8VuINx5CFo1t2WoecUjsCEIRyYt79 kmPn0GXo7Kr7HKct4ROYSGZeiuC6lzVQG1fffmEpgTk6oTzvT6PcpuP2L.x2EYVLfLsOYQcqX60CK6 xOs4wY6wRtiAXQ5njIZKEEB1guAHV4-N6uC87H0eskS-33uC7KE3m6Vo9tF0FQ9szA ) 
